\section{Higher intraocular pressure is associated with leukoaraiosis among middle-aged and elderly Koreans without glaucoma or dementia}

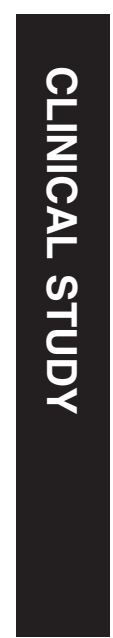

Eye (2014) 28, 715-719; doi:10.1038/eye.2014.63; published online 28 March 2014

Introduction

Ocular pressure is easily measured during a basic health examination. Elevated intraocular pressure is known to be a major, but modifiable, risk factor for glaucoma. ${ }^{1-3}$ In addition, high intraocular pressure has been associated with cardiovascular disease, metabolic syndrome, and neurodegenerative diseases, such as Alzheimer's disease. ${ }^{4-6}$

The term leukoaraiosis describes a pattern of diffuse white-matter abnormalities seen on brain magnetic resonance imaging (MRI) scans in the periventricular and subcortical white matter. ${ }^{7}$ Leukoaraiosis has long been regarded as a benign manifestation, with scarce or no clinical significance. However, leukoaraiosis is now drawing the interest of medical researchers, because it seems to be associated with a wide variety of geriatric health conditions, including gait disturbance, ${ }^{8}$ ischemic stroke, ${ }^{9}$ insulin resistance, and cognitive impairment. ${ }^{10}$ Thus early identification of high-risk leukoaraiosis groups is important from a gerontology perspective.

Although some previous studies reported that glaucoma was associated with overt cardiovascular and neurodegenerative diseases, to our knowledge, little is known about the relationship between intraocular pressure and leukoaraiosis in its preclinical stage. Therefore, this study aimed to examine the association between intraocular pressure and leukoaraiosis in middle-aged and elderly Koreans.
${ }^{1}$ Department of Family Medicine, CHA University School of Medicine, Seoul, Republic of Korea

${ }^{2}$ Department of Family Center, Yonsei University Wonju College of Medicine, Wonju, Republic of Korea

${ }^{3}$ Department of Family Medicine, Yonsei University College of Medicine, Seoul, Republic of Korea

Correspondence: Y-J Lee, Department of Family Medicine, Yonsei University College of Medicine, 225 Geumhakno, Cheoin-gu, Yongin-si, Gyeonggi-do 449-930, Republic of Korea Tel: +82 331 8710; Fax: +82 3315551 . E-mail: ukyjhome@yuhs.ac

Received: 24 May 2013 7 February 2014 Published online: 28 March 2014 Medicine, Health Promotion Accepted in revised form: 


\section{Materials and methods}

\section{Participants}

We retrospectively reviewed the medical records of 775 participants (486 men and 289 women) aged $>50$ years who voluntarily received a health examination, including brain MRI scans at the Health Promotion Center in Gangnam Severance Hospital, Seoul, Korea from January 2007 to November 2008. We excluded participants with a history of glaucoma or stroke. After this exclusion process, 753 Korean adults (474 men, 279 women) were included in the final analysis. The Institutional Review Board of Yonsei University College of Medicine, Seoul, Korea approved this study. We certify that all applicable institutional and governmental regulations concerning the ethical use of human volunteers were followed during this research.

\section{Data collection}

Medical staff performed all health examinations according to the standard procedures. Participants were asked about lifestyle behaviors, including cigarette smoking, alcohol consumption, and physical activity (more or less than two times per week), as well as whether they were currently undergoing treatment for any disease. If a participant was currently receiving treatment, they were asked for the date of diagnosis and a list of current medications. Trained staff reviewed the completed questionnaires and entered the responses into a database. Participants were classified as non-smokers, ex-smokers, or current smokers. Participants were categorized by their alcohol intake as either non-drinkers and abstainers (alcohol consumption $<140 \mathrm{~g}$ /week) or current drinkers (alcohol consumption $\geq 140 \mathrm{~g} /$ week). Body weight and height were measured in light indoor clothing without shoes to the nearest $0.1 \mathrm{~kg}$ and $0.1 \mathrm{~cm}$, respectively. Body mass index (BMI) was calculated as the ratio of weight $(\mathrm{kg}) /$ height $\left(\mathrm{m}^{2}\right)$. Blood pressure was taken after $5 \mathrm{~min}$ of rest using an automated device (TM-2665P, A\&D Co., Ltd., Tokyo, Japan).

After a 12-h overnight fast, blood samples were taken from the antecubital vein. Fasting plasma glucose, total cholesterol, triglycerides, and HDL-cholesterol were measured using the Hitachi 7600-110 chemistry autoanalyzer (Hitachi Co., Tokyo, Japan). Hypertension was defined as having a history of taking hypertension medication, systolic blood pressure $\geq 140 \mathrm{~mm} \mathrm{Hg}$, and / or diastolic blood pressure $\geq 90 \mathrm{~mm} \mathrm{Hg}$. Diabetes was defined as having a history of taking diabetes medication and/or a fasting plasma glucose level $\geq 7.0 \mathrm{mmol} / 1$.

The ophthalmological examinations were performed by ophthalmologists. Intraocular pressure was measured in each eye with a Goldmann applanation tonometer
(AT 900, Haag-Streit, Bern, Switzerland), and the mean of both eyes was used as the pressure variable.

A diagnosis of leukoaraiosis was based on brain MRI features with a 3.0 T MR scanner with a standard head coil (GE Signa VH/I; GE Medical Systems, Milwaukee, WI, USA). All participants were examined after administration of a Gd-DTPA contrast agent. The MRI protocol included the collection of $\mathrm{T}_{1}$ - and $\mathrm{T}_{2}$-weighted images, as well as fluid-attenuated inversion recovery (FLAIR) images. Two experienced radiologists who were unaware of the study aims and who were blinded evaluated all images. Leukoaraiosis was diagnosed by the presence of a hyperintensity on $\mathrm{T}_{2}$-weighted images or FLAIR without hypointensity on $\mathrm{T}_{1}$-weighted images. This assessment was done on precontrast images. The coefficients of variation for interoperator and intraoperator reproducibility were 6.4 and $4.3 \%$, respectively.

\section{Statistical analysis}

The demographic and biochemical characteristics of the study population according to the presence of leukoaraiosis were compared using the independent two-sample $t$-test for continuous variables and the chisquared test for categorical variables. A multiple logistic regression analysis was performed in order to determine whether intraocular pressure is an independent determinant for leukoaraiosis. All analyses were conducted using the SAS software version 9.1 (SAS Institute Inc., Cary, NC, USA). All statistical tests were two-sided, and the statistical significance was determined at $P<0.05$.

\section{Results}

Table 1 shows the general characteristics of the study population $(n=753)$. The overall prevalence of leukoaraiosis was $7.3 \%$. The mean age was $63.5 \pm 8.4$ years in the leukoaraiosis group and $57.4 \pm 6.3$ years in the non-leukoaraiosis group $(P<0.001)$. Mean intraocular pressure was significantly higher in participants with leukoaraiosis than in those without $(P=0.028)$. Systolic blood pressure and fasting plasma glucose levels, as well as the prevalence of hypertension and diabetes, were higher in the leukoaraiosis group.

Table 2 shows the risk of leukoaraiosis according to changes in intraocular pressure. After adjusting for age, sex, BMI, systolic blood pressure, fasting plasma glucose, total cholesterol, smoking status, alcohol consumption, physical activity, hypertension medication, and diabetes medication, the OR $(95 \% \mathrm{CI})$ for leukoaraiosis was 1.18 (1.06-1.31) for each $1 \mathrm{~mm} \mathrm{Hg}$ increase in intraocular pressure. 
Table 1 Participant characteristics, mean \pm SD or $\%$

\begin{tabular}{lcccc}
\hline & Total $(\mathrm{n}=753)$ & Leukoaraiosis $(\mathrm{n}=55)$ & Non-leukoaraiosis $(\mathrm{n}=698)$ & P-value \\
\hline Age, years & $57.8 \pm 6.6$ & $63.5 \pm 8.4$ & $57.4 \pm 6.3$ & $<3.1$ \\
Female, $\%$ & 37.1 & 36.4 & $23.8 \pm 2.7$ & 0.912 \\
Body mass index, $\mathrm{kg} / \mathrm{m}^{2}$ & $23.8 \pm 2.7$ & $23.7 \pm 2.7$ & 18.8 & 0.744 \\
Current smoker, $\%$ & 18.2 & 10.9 & 16.3 & 0.145 \\
Alcohol consumption, $\%$ & 16.3 & 16.4 & 61.0 & 0.995 \\
Regular exercise, \% & 61.2 & 63.6 & $123.7 \pm 16.0$ & 0.702 \\
Systolic blood pressure, $\mathrm{mm} \mathrm{Hg}$ & $124.1 \pm 16.2$ & $728.8 \pm 17.6$ & $77.5 \pm 9.6$ & 0.024 \\
Diastolic blood pressure, $\mathrm{mm} \mathrm{Hg}$ & $77.6 \pm 9.7$ & $6.0 \pm 1.8$ & $5.5 \pm 1.3$ & 0.087 \\
Fasting plasma glucose, $\mathrm{mmol} / 1$ & $5.5 \pm 1.3$ & $5.1 \pm 1.2$ & $5.1 \pm 0.9$ & 0.040 \\
Total cholesterol, mmol/1 & $5.1 \pm 0.9$ & $1.3 \pm 0.3$ & $1.3 \pm 0.3$ & 0.603 \\
HDL-cholesterol, mmol/1 & $1.3 \pm 0.3$ & $1.6 \pm 0.9$ & $1.4 \pm 0.8$ & 0.800 \\
Triglyceride, mmol/1 & $1.4 \pm 0.8$ & $14.3 \pm 2.9$ & 36.5 & 0.209 \\
Intraocular pressure, mm Hg & $13.5 \pm 2.6$ & 69.1 & 7.0 & 0.028 \\
Hypertension, $\%$ & 38.9 & 20.4 & $<0.001$ \\
Diabetes, $\%$ & 8.0 & & $<0.001$ \\
\hline
\end{tabular}

Table 2 Adjusted odds ratio for leukoaraiosis as a dependent variable and the associated factors as independent variables in middle-aged and elderly Koreans

\begin{tabular}{lcc}
\hline & OR (95\% CIs) & P-value \\
\hline Age, years & $1.12(1.08-1.17)$ & $<0.001$ \\
Female gender & $0.96(0.49-1.89)$ & 0.907 \\
Current smoking, yes or not & $0.55(0.21-1.43)$ & 0.219 \\
Alcohol consumption, $\geq 140 \mathrm{~g} /$ week or less & $1.64(0.71-3.78)$ & 0.246 \\
Regular exercise, $\geq$ twice $/$ week or less & $1.13(0.60-2.10)$ & 0.712 \\
Body mass index, $\mathrm{kg} / \mathrm{m}^{2}$ & $0.96(0.86-1.08)$ & 0.524 \\
Systolic blood pressure, $\mathrm{mm} \mathrm{Hg}$ & $1.00(0.98-1.02)$ & 0.837 \\
Fasting plasma glucose, $\mathrm{mmol} / \mathrm{l}$ & $1.13(0.88-1.44)$ & 0.351 \\
Total cholesterol, mmol/1 & $1.37(1.00-1.87)$ & 0.053 \\
Hypertension medication, yes or no & $2.52(1.34-4.76)$ & 0.004 \\
Diabetes medication, yes or no & $1.37(0.48-3.93)$ & 0.559 \\
Intraocular pressure, mm Hg & $1.18(1.06-1.31)$ & 0.002 \\
\hline
\end{tabular}

\section{Discussion}

We examined the association of intraocular pressure with leukoaraiosis in middle-aged and elderly Koreans without glaucoma or dementia. Higher intraocular pressure was associated with an increased prevalence of leukoaraiosis. This association remained valid after adjusting for the medication history of drugs that could modify vascular function parameters, such as antihypertensive or antidiabetic drugs. These findings indicate that higher intraocular pressure may be closely related with leukoaraiosis, independently of cardiometabolic risk factors. To the best of our knowledge, this is the first study to shed light on the association between leukoaraiosis risk and increased intraocular pressure.

Leukoaraiosis is a manifestation of ischemic injury in white-matter substances that results from chronic ischemia due to age-related cerebral small vessel pathology. ${ }^{11}$ In fact, postmortem studies have indicated that leukoaraiosis seen on brain MRI scans is associated with degenerative changes in arterioles that are related to atherosclerosis and with lipohyalinosis of white matter that perforates small arteries. ${ }^{12,13}$

Some plausible mechanisms that link increased intraocular pressure with risk for leukoaraiosis deserve consideration. High intraocular pressure and leukoaraiosis share some of the same pathogenic factors with atherosclerosis, such as endothelial dysfunction and atherothrombosis. Intraocular pressure and leukoaraiosis have also been positively associated with clustered cardiometabolic risk factors and metabolic syndrome. ${ }^{14-16}$ Atherosclerotic changes in ocular vessels and the cerebral artery that are associated with cardiometabolic risk factors may be responsible for elevated intraocular pressure and leukoaraiosis. ${ }^{17,18}$ Indeed, in the present study, hypertension and diabetes mellitus were more prevalent in the leukoaraiosis group. Atherosclerotic changes in the ocular vessels and cerebral artery have been simultaneously observed in patients with glaucoma. ${ }^{19,20}$ In a condition of elevated intraocular pressure, incident risk of spontaneous platelet aggregation has been found to be high, independently of cardiovascular diseases. ${ }^{21}$ Furthermore, the significant association between intraocular pressure and leukoaraiosis may be linked to an altered platelet aggregation. In the Cardiovascular Health Study, Fornage $e t a l^{22}$ reported that inflammatory markers such as interleukin-6 and C-reactive protein were significantly associated with the presence of a white-matter lesion. These pro-inflammatory cytokines may stimulate the bone marrow to produce large platelets that, in turn, accelerate the progression of ischemic atherothrombosis in ocular vessels. ${ }^{23}$

As described earlier, previous research has also reported that leukoaraiosis is closely associated with chronic endothelial dysfunction, ${ }^{24}$ which may lead to increased levels of vascular endothelial growth factor 
(VEGF) and von Willebrand factor (vWF). ${ }^{25}$ Lip et al ${ }^{26}$ demonstrated that abnormal vascular permeability by VEGF and vWF is associated with glaucoma. Moreover, dysregulation of the autonomic nervous system such as the hyperactivation of the ocular sympathetic nerve increases intraocular pressure as well as vascular dysfunction..$^{25,27}$

Our study has some limitations. First, we used a cross-sectional design, and additional studies are needed to establish the cause and effect between intraocular pressure and leukoaraisos. Second, the study population may not represent the general Korean population, because the study participants were volunteers who visited a single hospital for a health check-up and appeared to be healthy individuals. Therefore, this study may have been affected by selection bias. In this regard, we speculate that current smoking status and BMI were not associated with an increased prevalence of leukoaraiosis. Third, we used a binary classification for leukoaraiosis without using a severity scale, because a severity scale was not adopted at the beginning of this study. Further research is warranted to clarify the relationship of intraocular pressure with severity of leukoaraiosis. Finally, we have not considered some additional confounding factors such as cataract surgery, heart disease, or peripheral vascular disease, because the current study was based on the retrospective review. Therefore, the strength of association between intraocular pressure and leukoaraiosis might be overestimated.

In conclusion, intraocular pressure was found to be independently and positively associated with leukoaraiosis, regardless of classical cardiometabolic risk factors in middle-aged and elderly Koreans without glaucoma or dementia. This indicates that higher intraocular pressure may be an useful additional measure in assessing the risk of leukoaraiosis in the clinical setting.

\section{Summary}

\section{What was known before}

- High intraocular pressure is associated with cardiovascular disease and metabolic syndrome.

- Leukoaraiosis is a manifestation of ischemic injury in brain white-matter substances that results from chronic ischemia.

- Leukoaraiosis has been associated with cognitive impairment and ischemic stroke.

\section{What this study adds}

- Intraocular pressure was found to be positively associated with leukoaraiosis.

- Higher intraocular pressure may be a useful additional measure in assessing the risk of leukoaraiosis.

\section{Conflict of interest}

The authors declare no conflict of interest.

\section{References}

1 Anderson DR. Glaucoma: the damage caused by pressure XLVI Edward Jackson memorial lecture. Am J Ophthalmol 1989; 108: 485-495.

2 Leske MC, Connell AM, Wu SY, Hyman LG, Schadchat AP. Risk factors for open-angle glaucoma. The Barbados Eye Study. Arch Ophthalmol 1995; 113: 918-924.

3 Goldberg I. Relationship between intraocular pressure and preservation of visual field in glaucoma. Surv Opthalmol 2003; 48(Suppl 1): S3-S7.

4 Shiose S. Intraocular pressure: new perspectives. Surv Ophthalmol 1990; 34: 413-435.

5 Oh SW, Lee SY, Park CY, Kim DJ. Elevated intraocular pressure is associated with insulin resistance and metabolic syndrome. Diabetes Metab Res Rev 2005; 21: 434-440.

6 Bayer AU, Ferrari F, Erb C. High occurrence rate of glaucoma among patients with Alzheimer's disease. Eur Neurol 2002; 47: 165-168.

7 Vernooij MW, Ikram MA, Tanghe HL, Vincent AJ, Hofman A, Krestin GP et al. Incidental findings on brain MRI in the general population. N Engl J Med 2007; 357: 1821-1828.

8 Starr JM, Leaper SA, Murray AD, Lemmon HA, Staff RT, Deary IJ et al. Brain white matter lesions detected by magnetic resonance imaging are associated with balance and gait speed. J Neurol Neurosurg Psychiatr 2003; 74: 94-98.

9 Wiszniewska M, Devuyst G, Bogousslavsky J, Ghika J, van Melle G. What is the significance of leukoaraiosis in patients with acute ischemic stroke? Arch Neurol 2000; 57: 967-973

10 Vermeer SE, Prins ND, Den Heijer T, Hofman A, Koudstaal PJ, Breteler MM. Silent brain infarcts and the risk of dementia and cognitive decline. N Engl J Med 2003; 348: 1215-1222.

11 Pantoni L, Garcia JH. Pathogenesis of leukoaraiosis: a review. Stroke 1997; 28: 652-659.

12 Brun A, Englund E. A white matter disorder in dementia of the Alzheimer type: a pathoanatomical study. Ann Neurol 1986; 19: 253-262.

13 Brown WR, Moody DM, Challa VR, Thore CR, Anstrom JA. Venous collagenosis and arteriolar tortuosity in leukoaraiosis. J Neurol Sci 2002; 203-204: 159-163.

14 Wu SY, Leske MC. Associations with intraocular pressure in the Barbados. Eye Study Arch Ophthalmol 1997; 115: 1572-1576.

15 Lee JS, Lee SH, Oum BS, Chung JS, Cho BM, Hong JW. Relationship between intraocular pressure and systemic health parameters in a Korean population. Clin Experiment Ophthalmol 2002; 30: 237-241.

16 Chang YC, Lin J-W, Wang LC, Chen HM, Hwang JJ, Chuang LM. Association of intraocular pressure with the metabolic syndrome and novel cardiometabolic risk factors. Eye 2010; 24: 1037-1043.

17 Choi HS, Cho YM, Kang JH, Shin CS, Park KS, Lee HK. Cerebral white matter hyperintensity is mainly associated with hypertension among the components of metabolic syndrome in Koreans. Clin Endocrinol 2009; 71: 184-188.

18 Park K, Yasuda N, Toyonaga S, Yamada SM, Nakabayashi H, Nakasato M. Significant association between leukoaraiosis and metabolic syndrome in healthy subjects. Neurology 2007; 69: 974-978. 
19 Elwyn H. Calcified carotid artery with atrophy of the optic nerve, cupping, and low tension. Arch Ophthalmol 1940; 24: 476-478.

20 McLean JM, Ray BS. Soft glaucoma and calcification on the internal carotid arteries. Arch Ophthalmol 1947; 38: 154-158.

21 Hoyng PF, Greve EL, Frederikse K, Geijssen C, Oosting H. Platelet aggregation and glaucoma. Doc Ophthalmol 1985; 61 : 167-173.

22 Fornage M, Chiang A, O'Meara ES, Psaty BM, Reiner AP, Siscovick DS et al. Biomarkers of inflammation and MRI-defined small vessel disease of the brain: the Cardiovascular Health Study. Stroke 2008; 39: 1952-1959.

23 Vizioli L, Muscari S, Muscari A. The relationship of mean platelet volume with the risk and prognosis of cardiovascular diseases. Int J Clin Pract 2009; 63: 1509-1515.
24 Hassan A, Hunt BJ, O'Sullivan M, Parmar K, Bamford JM, Briley $\mathrm{D}$ et al. Markers of endothelial dysfunction in lacunar infarction and ischaemic leukoaraiosis. Brain 2003; 126: 424-432.

25 Pache M, Flammer J. A sick eye in a sick body? Systemic findings in patients with primary open-angle glaucoma. Surv Ophthalmol 2006; 51: 179-212.

26 Lip PL, Felmeden DC, Blann AD, Matheou N, Thakur S, Cunliffe IA et al. Plasmavascular endothelial growth factor, solubleVEGF receptorFLT-1, and von Willebrand factor in glaucoma. Br J Ophthalmol 2002; 86: 1299-1302.

27 Belmonte C, Bartels SP, Liu JH, Neufeld AH. Effects of stimulation of the ocular sympathetic nerves on IOP and aqueous humor flow. Invest Ophthalmol Vis Sci 1987; 28: 1649-1654. 